\title{
Circadian rhythm phase shifts caused by timed exercise vary with chronotype
}

\author{
J. Matthew Thomas, ${ }^{1,2}$ Philip A. Kern, ${ }^{2,3,4}$ Heather M. Bush, ${ }^{2,5}$ Kristen J. McQuerry, ${ }^{5}$ W. Scott Black, ${ }^{6}$ \\ Jody L. Clasey, ${ }^{1,2,4}$ and Julie S. Pendergast ${ }^{2,4,7,8}$ \\ 'Department of Kinesiology and Health Promotion, ${ }^{2}$ Center for Clinical and Translational Science, ${ }^{3}$ The Department \\ of Internal Medicine, Division of Endocrinology, ${ }^{4}$ Barnstable Brown Diabetes and Obesity Center, ${ }^{5}$ Department of \\ Biostatistics, ${ }^{6}$ Department of Clinical Sciences, ${ }^{7}$ Department of Biology, and ${ }^{8}$ Saha Cardiovascular Research Center, \\ University of Kentucky, Lexington, Kentucky, USA.
}

BACKGROUND. The circadian system entrains behavioral and physiological rhythms to environmental cycles, and modern lifestyles disrupt this entrainment. We investigated a timed exercise intervention to phase shift the internal circadian rhythm.

METHODS. In 52 young, sedentary adults, dim light melatonin onset (DLMO) was measured before and after 5 days of morning ( 10 hours after DLMO; $\boldsymbol{n}=26$ ) or evening (20 hours after DLMO; $\boldsymbol{n}=\mathbf{2 6}$ ) exercise. Phase shifts were calculated as the difference in DLMO before and after exercise.

RESULTS. Morning exercise induced phase advance shifts ( $0.62 \pm 0.18$ hours) that were significantly greater than phase shifts from evening exercise $(-0.02 \pm 0.18$ hours; $P=0.01)$. Chronotype also influenced the effect of timed exercise. For later chronotypes, both morning and evening exercise induced phase advances ( $0.54 \pm 0.29$ hours and $0.46 \pm 0.25$ hours, respectively). In contrast, earlier chronotypes had phase advances from morning exercise ( $0.49 \pm 0.25$ hours) but had phase delays from evening exercise $(-0.41 \pm 0.29$ hours $)$.

CONCLUSION. Late chronotypes - those who experience the most severe circadian misalignment - may benefit from phase advances induced by exercise in the morning or evening, but evening exercise may exacerbate circadian misalignment in early chronotypes. Thus, personalized exercise timing prescription, based on chronotype, could alleviate circadian misalignment in young adults.

TRIAL REGISTRATION. Trial registration can be found at www.clinicaltrials.gov (NCT04097886).

FUNDING. Funding was supplied by NIH grants UL1TR001998 and TL1TR001997, the Barnstable Brown Diabetes and Obesity Center, the Pediatric Exercise Physiology Laboratory Endowment, the Arvle and Ellen Turner Thacker Research Fund, and the University of Kentucky.

Authorship note: JLC and JSP contributed equally to this work.

Conflict of interest: The authors have declared that no conflict of interest exists.

Copyright: () 2020, American Society for Clinical Investigation.

Submitted: October 14, 2019 Accepted: December 19, 2019 Published: February 13, 2020

Reference information: /CI Insight. 2020;5(3):e134270.

https://doi.org/10.1172/jici.

insight.134270.

\section{Introduction}

The circadian system controls 24-hour cycles of behavior and physiology, such as rest-activity and feeding rhythms, and has evolved to entrain to natural cycles of light and dark (1). This synchronization between internal and external rhythms has been shown to improve fitness in model organisms (2-4). However, in the 24/7 Western society, most individuals no longer have exclusive exposure to the natural light/dark cycle. People are active and have light exposure long after sunset, they eat late into the night, and they wake up to alarm clocks hours before they would wake naturally $(5,6)$. This has resulted in a society that is living in a chronic state of circadian disruption.

Numerous studies have shown that circadian disruption is a health risk. Shift work chronically disrupts the circadian system and is also associated with increased risk of cancer, obesity, and cardiovascular disease (6-11). Nonshift workers also experience chronic forms of circadian disruption. A total of $70 \%$ of the population experiences social jetlag, which is a misalignment between social obligations and internal circadian rhythms (e.g., waking up to an early-morning alarm clock) (6). Social jetlag is associated with increased cortisol levels and resting heart rate, insulin resistance, obesity, metabolic syndrome, and systemic inflammation $(6,9,12-14)$. People with a late chronotype ("night owls") often experience 
social jetlag because they have early-morning work schedules that do not match their natural internal rhythms. People with a late chronotype are less physically active, have a higher BMI, and are at increased risk of developing type 2 diabetes and metabolic syndrome (15-18). Misalignment of circadian rhythms may be a contributing factor leading to obesity and metabolic dysfunction in late chronotypes (19).

The circadian system is remarkably sensitive to environmental cues that synchronize internal rhythms to external cycles. Therefore, behavioral, noninvasive therapeutics could tap into these input circuits and align internal and external rhythms in individuals with disrupted circadian systems. In addition to light, exercise can entrain the circadian system in mammals (20-24). It is well known that regular physical activity has substantial health benefits, including improvements in cardiometabolic health, obesity, anxiety, depression, cognition, and overall wellbeing (25-30). However, the role of exercise in regulating the circadian system has not been studied sufficiently. Exercise, if performed at the appropriate time of day, could shift the phase of the internal circadian rhythm and therefore improve circadian misalignment. In many individuals, especially in people with a late chronotype, an advance of the internal circadian rhythm would better align internal rhythms with the environment and with standard social schedules. Recent studies in mice showed that exercise performance, gene transcription, and energy utilization depend on the time of day of exercise $(31,32)$. Clinical studies have conflicting results, but some have shown that morning or early afternoon exercise phase advances, while evening exercise phase delays the internal circadian rhythm (21, 33-36). However, most of these prior studies were performed in controlled lab conditions, and many studies included physically active adults such that the results may have been confounded by recent nonlaboratory exercise regimens.

The purpose of this study was to investigate the impact of timed exercise on circadian rhythms in free-living (i.e., not in the laboratory), sedentary young adults. We found that morning (compared with evening) exercise advanced the phase of the circadian rhythm and that both morning and evening exercise advanced circadian phase in people with a late chronotype.

\section{Results}

Time of exercise affects the phase of circadian rhythms in young adults. To investigate the effects of timed exercise on phase shifts of the internal circadian rhythm, we studied young men $(n=16)$ and women $(n=36)$ (Figure 1, Table 1, Supplemental Table 1, Supplemental Table 2, and Supplemental Table 3; supplemental material available online with this article; https://doi.org/10.1172/jci.insight.134270DS1). Young adults tend to have later sleep timing (late chronotype) (37), and they could therefore have the greatest benefit from timed exercise, if that exercise advanced their circadian rhythms. We also studied only sedentary participants ( $\leq 2$ hour moderate-vigorous structured exercise/week), and they exercised only during the 30-minutes scheduled and supervised session. Age, anthropometric, body composition, and cardiorespiratory fitness measures did not differ between participants who exercised in the morning compared with those who exercised in the evening (Table 1 and Supplemental Table 1).

To mark the phase of the internal circadian rhythm and to time the exercise intervention, we measured the rise in salivary melatonin levels before habitual bedtime, called the dim light melatonin onset (DLMO), which is a well-established, reliable, and widely used marker of internal circadian phase of human participants (38). A baseline DLMO was performed first (Supplemental Figure 1). After the baseline DLMO, participants were then randomized to an exercise intervention at either 10 hours after DLMO ("morning exercise") or 20 hours after DLMO ("evening exercise"). We then measured DLMO again after 5 days of the timed exercise intervention to determine if there were changes in phase. In baseline and postexercise DLMOs, salivary melatonin levels were initially at low daytime levels $(<2 \mathrm{pg} / \mathrm{mL})$ at the onset of the DLMO protocol and then increased past the $4 \mathrm{pg} / \mathrm{mL}$ threshold. In 2 of 52 participants, however, salivary melatonin levels were variable during the first 2 hours of the DLMO protocol.

After adjusting for the difference in baseline DLMO between the groups, the morning exercisers had a significantly greater phase advance $(0.62 \pm 0.18$ hours $)$ than evening exercisers $(-0.02 \pm 0.18$ hours; Figure 2; $P=0.01$, phase shift $\mathrm{DLMO}_{\text {adjusted }}$ in Table 2; Supplemental Table 4). Importantly, the morning exercisers had a 0.6 -hour phase advance, which could theoretically better align their internal circadian rhythms with the light-dark cycle and with early-morning social obligations.

We also analyzed sleep during the 5 days of exercise using actigraphy. Sleep onset and midsleep during the exercise days were significantly earlier in the morning group compared with the evening group (Table 2). This is consistent with the trending earlier chronotype in this group, and it may also reflect a phase advance caused by morning exercise. Sleep fragmentation index, a measure of sleep quality, and sleep 


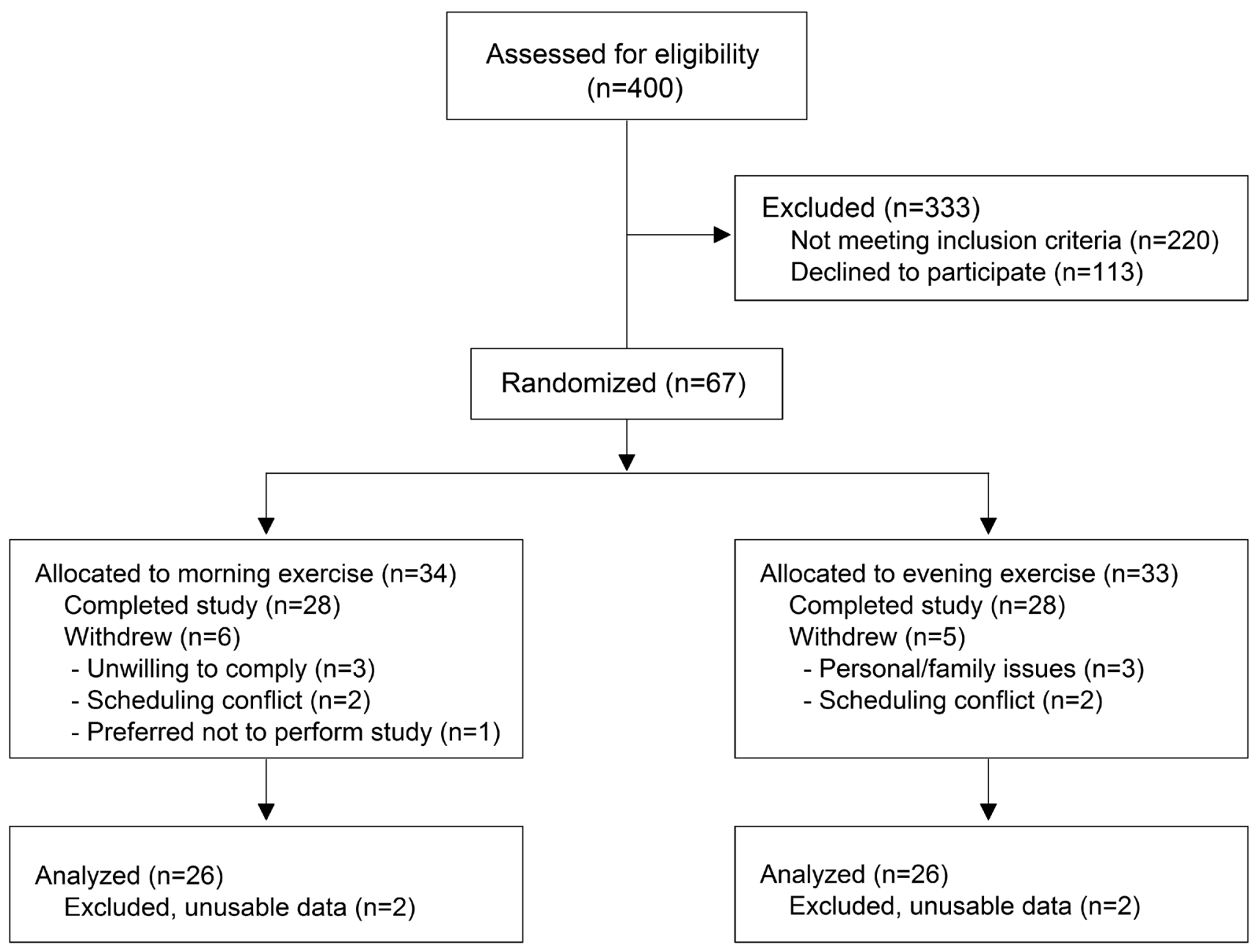

Figure 1. CONSORT diagram for this randomized trial. Sixty-seven participants were randomized to morning or evening exercise, and 11 participants subsequently withdrew. Fifty-two participants were included in the analytic data set (after removing 4 participants because phase could not be determined from the melatonin data).

duration did not differ between the exercise groups. Therefore, despite the earlier timing of sleep in the morning exercise group, the quality and duration of sleep were similar between groups.

The magnitudes of phase shifts and other sleep and circadian parameters were not significantly different between male and female participants (Supplemental Table 2). Consistent with previous studies, there were sex differences in anthropometric, body composition, and cardiorespiratory fitness measures (Supplemental Table 2) $(39,40)$.

Phase shifts caused by timed exercise depend on chronotype. For this study, we enrolled young, sedentary participants without regard to chronotype. As a result, our participants had variable chronotypes that were normally distributed, similar to previous studies of chronotype in young adults (41-43) (Supplemental Figure 1). We next determined whether the magnitude of phase shifts elicited by timed exercise depended on chronotype. We found that there was no significant association between DLMO and phase shifts in the morning exercise group (Figure 3A), but there was a strong association between chronotype and phase shifts in the evening exercise group (Figure 3B) such that late chronotypes had the greatest phase advance. Because these analyses suggested that baseline internal phase modifies the relationship between timed exercise and phase shift, we examined the impact of morning or evening exercise on phase shift stratified by earlier and later chronotypes (Figure 4 and Supplemental Table 5). We found that earlier chronotypes had phase advances $(0.49 \pm 0.25$ hours) with morning exercise but phase delays $(-0.41 \pm 0.29$ hours) with evening exercise. In contrast, later chronotypes had phase advances with either morning $(0.54 \pm 0.29$ hours) or evening $(0.46 \pm 0.25$ hours) exercise. 
Table 1. Characteristics of study participants

\begin{tabular}{|c|c|c|c|}
\hline Variable $^{A}$ & Morning group $\boldsymbol{N}=\mathbf{2 6}$ (range) & Evening group $N=26$ (Range) & $P$ value \\
\hline Age & $\begin{array}{c}24.77 \pm 1.20 \\
(18-45 \text { years })\end{array}$ & $\begin{array}{l}23.73 \pm 0.96 \\
(18-35 \text { years })\end{array}$ & 0.50 \\
\hline BMI $\left(\mathrm{kg} / \mathrm{m}^{2}\right)$ & $\begin{array}{c}24.12 \pm 0.85 \\
(16.61-36.91)\end{array}$ & $\begin{array}{l}24.56 \pm 0.85 \\
(17.13-36.20)\end{array}$ & 0.72 \\
\hline \multicolumn{4}{|l|}{$\begin{array}{l}\text { Anthropometric and body } \\
\text { composition }\end{array}$} \\
\hline Body fat percentage (\%) & $\begin{array}{c}31.11 \pm 1.63 \\
(21.40-48.60)\end{array}$ & $\begin{array}{c}32.45 \pm 1.81 \\
(12.40-50.40)\end{array}$ & 0.58 \\
\hline Mineral-free lean mass (kg) & $\begin{array}{c}42.17 \pm 1.46 \\
(29.83-57.86)\end{array}$ & $\begin{array}{c}42.45 \pm 1.36 \\
(30.58-60.73)\end{array}$ & 0.89 \\
\hline \multicolumn{4}{|l|}{ Cardiorespiratory fitness } \\
\hline $\mathrm{VO}_{2 \text { peak }}(\mathrm{mL} / \mathrm{kg} / \mathrm{min})$ & $\begin{array}{c}38.91 \pm 1.55 \\
(24.20-55.80)\end{array}$ & $\begin{array}{c}36.47 \pm 1.70 \\
(21.60-52.50)\end{array}$ & 0.29 \\
\hline
\end{tabular}

${ }^{A}$ Data are presented as mean \pm SEM. ${ }^{B}$ Baseline participant characteristics for the morning and evening exercise groups were compared using independent sample $t$-tests.

\section{Discussion}

Exercise has many well-established benefits to physical and mental health (25-30). The intensity, duration, frequency, mode, volume, and progression of exercise to optimize the beneficial effects have been well characterized $(26,44,45)$. However, the proper timing of exercise and its potential added benefit of improving circadian entrainment have not been investigated thoroughly. Disruption of circadian rhythms (e.g., by shift work, social jetlag, early-morning schedules) is associated with metabolic disorders, cardiovascular disease, and cancer (6-11). If exercise could reduce circadian disruption, then it may also improve the risk factors associated with this disruption.

A tenet of the mammalian circadian system is that it is differentially sensitive to stimuli given at different times of day. Therefore, it is likely that the response of the internal circadian rhythm to exercise depends on the time of day of exercise. We chose morning and evening exercise for 2 reasons. First, morning exercise has been shown, in some studies, to cause significant phase advances, which could alleviate circadian misalignment in late chronotypes and others whose internal rhythms are delayed relative to the environment $(21,33,34)$. Second, morning and evening are the most common times when people exercise on weekdays, so exercise at these times could be implemented as feasible behavioral interventions for circadian disruption in free-living individuals (46).

When all participants were considered, we found that morning exercise advanced the phase of the internal circadian rhythm, while evening exercise did not in young sedentary adults. Young adults, on average, have the latest chronotypes and, therefore, the greatest potential of all ages to experience circadian disruption caused by misalignment of their (later) internal circadian rhythms with early-morning obligations (37). Therefore, our study showed that morning exercise has the greatest potential to alleviate circadian misalignment in young adults. With regard to the feasibility of implementing a morning exercise regimen in a young adult, the exercise timing in this study was based on DLMO; therefore, a person with a late chronotype, as is often seen in young people, would likely be exercising midmorning or late morning. Thus, a morning exercise prescription is feasible even for a person with a late chronotype.

The mechanisms by which morning exercise advances the internal circadian rhythm are unknown. It is well known that exposure to morning light can phase advance the internal rhythm $(47,48)$; therefore, the combination of exposure to morning light and morning exercise could have additive phase-advancing effects on young adults in free-living conditions. Indeed, a previous study performed in controlled laboratory conditions that combined the 2 stimuli observed an additive phase-shifting effect (20). Thus, the combination of light and exercise may be a very effective treatment strategy to alleviate social jetlag and the associated metabolic derangements.

Although morning exercise was the ideal time to induce phase advances in the group as a whole, we also found that baseline DLMO influenced the phase advance caused by timed exercise. For later chronotypes, both morning and evening exercise advanced the internal circadian rhythm. In contrast, earlier chronotypes had 


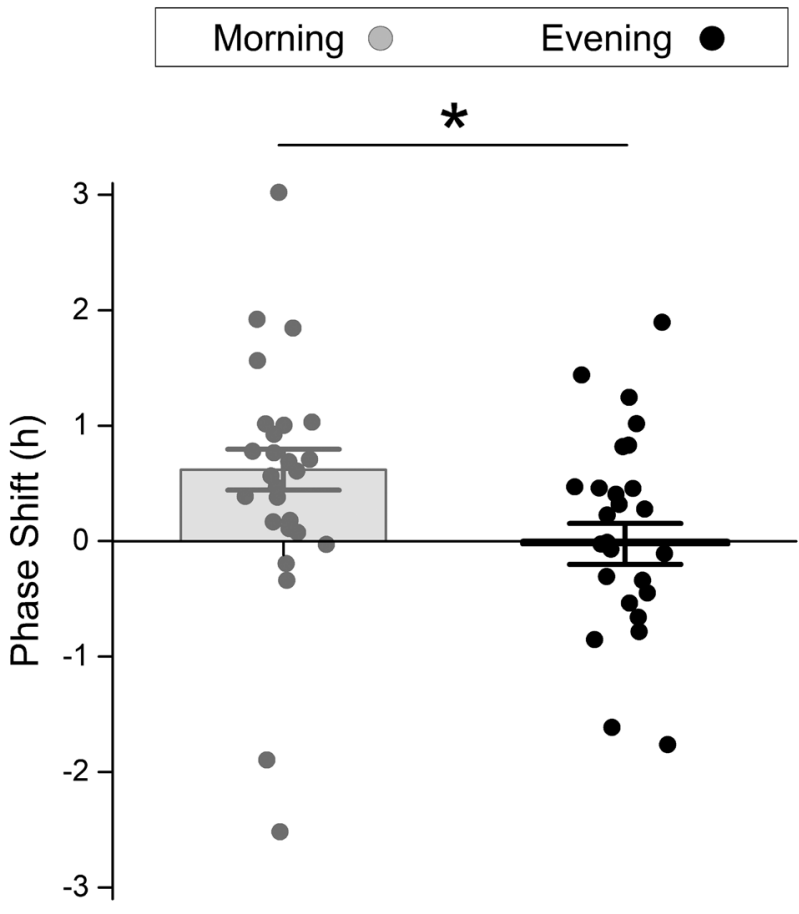

Figure 2. Morning exercise advances the phase of the internal circadian rhythm. Young sedentary adults exercised for 5 days either in the morning ( $n=26$ ) or evening $(n=26)$. Phase shift was calculated as the difference in the timing of DLMO before and after 5 days of exercise and was adjusted for the difference in baseline DLMO between the groups. Data points are raw data. Bars are mean \pm SEM from the 1-way ANCOVA model including exercise group and DLMO. ${ }^{*} P=0.01$

phase advances when they exercised in the morning, but they had phase delays when they exercised in the evening. These results suggest the need for personalized exercise timing prescriptions based on chronotype. Late chronotypes, who experience the most severe circadian misalignment, may benefit from exercise in the morning or evening. On the other hand, evening exercise may exacerbate circadian misalignment in early chronotypes.

Numerous epidemiological studies have also shown that cumulative years of exposure to circadian disruption (e.g., >5 years) markedly increases the risk of heart disease, breast cancer, and type 2 diabetes compared with acute exposure (49-51). Since the negative effects of circadian disruption increase with years of exposure, it is ideal to target young adults, who are particularly susceptible to circadian misalignment, to reduce the cumulative years of exposure. Our study demonstrates that timed exercise is effective in shifting the phase of the internal circadian rhythm and, thus, has the potential to reduce early-life exposure to circadian disruption and thereby reduce cumulative exposure over a lifetime.

The design of this study had a number of strengths when compared with previous studies. This study involved participants in "free-living conditions," and the results of our study were in agreement with previous studies in laboratory conditions where morning/early afternoon exercise similarly advanced the phase of the internal circadian rhythm $(21,34)$. In addition, the exercise intensity was based on each participant's maximal graded exercise test $\left(\mathrm{GXT}_{\max }\right)$ results to provide standardized exercise stimuli and our exercise regimen was practical (moderate exercise for 30 minutes per day). This is in contrast to several previous studies that did not standardize the exercise intensity relative to cardiorespiratory fitness $(21,52)$, or used long (3-hour) exercise bouts or multiple exercise sessions per day (22, 53-55). We also chose exercise times that are common in daily routines. Morning and evening are the most accessible times for much of the population. Other studies have investigated the effects of exercise at all times throughout the day and even in the middle of the night, which are not practical for translation to free-living people $(20-22,33,36,53$, 56). Some previous studies also performed postexercise phase assessments on the same day as the exercise stimulus, making it difficult to differentiate between an acute effect of exercise or a phase shift $(36,54)$. In our study, we measured the phase of the internal circadian rhythm on the day after the 5th day of exercise, thereby avoiding the acute masking effect of the exercise session. Finally, although we used well-established and reliable measures of circadian rhythms (DLMO, actigraphy) and cardiorespiratory fitness ( $\mathrm{GXT}_{\max }$ ), and although we normalized exercise intensity and timing across participants to rigorously test the effect of timed exercise on circadian phase, we can also make these predictions and prescriptions using cheaper and easier methods. For example, we can predict internal phase (and therefore prescribe exercise time) from simple questionnaires (e.g., DLMO is highly correlated with preferred sleep timing) (57). 
Table 2. Circadian and sleep characteristics of study participants

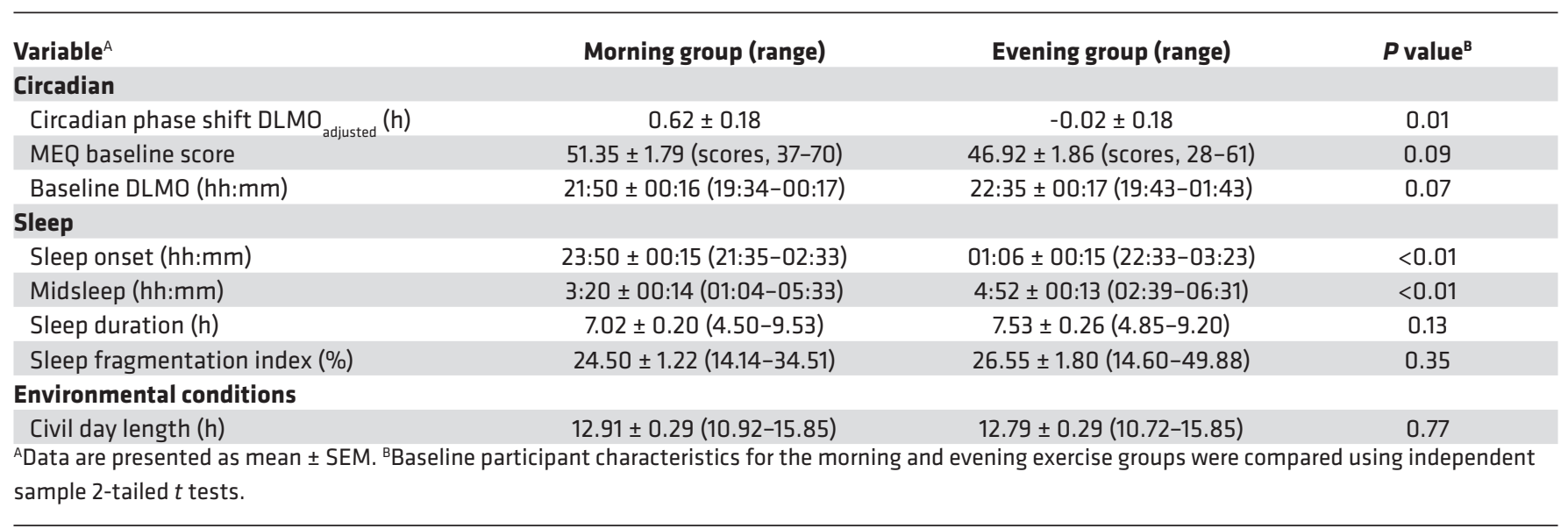

There were some limitations of our study. First, given that we designed our experiment to examine individuals under free-living conditions, we did not control for other stimuli that could affect the internal circadian phase. For example, travel to the lab for morning exercise could have resulted in increased light exposure during this time, facilitating phase advances independent of or in addition to exercise. In addition, the phase delays experienced by early chronotypes in the evening exercise group may have caused later wake times and deprived this group of morning light exposure. However, we did not design the study to collect baseline sleep measures; therefore, we could not determine whether the exercise intervention affected sleep timing. We began actigraphy recording at the time of consent and continuously recorded for the duration of the study. However, the number of baseline days recorded varied among the participants (1-9 days). Therefore, we could analyze sleep only during the exercise intervention.

A second limitation of our study is that we did not control for sleep timing or duration (e.g., individuals were instructed to sleep as they normally would). This was because our goal was to study participants in free-living conditions. Previous research has shown that changing sleep timing can affect DLMO (57, 58), but no studies, to our knowledge, have studied the effects of sleep duration/restriction on circadian phase shifts induced by exercise. It is possible that waking and traveling to the study site for morning exercise could have reduced sleep duration or sleep timing in this group compared with baseline. Since we did not measure baseline sleep, we cannot address whether the time of exercise affected sleep. However, there was no significant difference in sleep duration between morning and evening exercise groups during the 5 days of exercise, so it is unlikely that sleep duration markedly affected phase shifts.

A third limitation is that we studied only young, sedentary, healthy adults in free-living conditions. Therefore, it is unknown whether timed exercise will similarly phase shift the circadian system of older adults or of individuals with chronic illnesses (e.g., heart disease, diabetes). A previous study found that the phase-shifting effects of exercise were similar in younger and older adults (59), so we can speculate that timed exercise may similarly affect older adults. However, while we propose that a phase advance is typically the ideal shift for young adults (because their internal rhythm typically lags behind their social obligations), a phase delay may be ideal for older adults. This is because circadian phase advances as a normal process of aging $(60,61)$. Therefore, older adults often wake up early in the morning before their social obligations, and a phase delay could allow them to sleep later. Neurodegenerative diseases, such as Alzheimer's disease (62), also have comorbid circadian disruption, and future research could investigate the efficacy of timed exercise in reducing circadian disruption in chronically ill participants.

In this study, we only investigated the effect of moderate cardiovascular exercise on circadian phase. It is possible that other exercise modalities (e.g., resistance training) may also phase shift circadian rhythms. However, to our knowledge, only cardiovascular exercise has been used in previous studies (20, 22, 34, 36, 56). Exercise duration and intensity may influence phase shifts. A previous study found that a low-intensity, 3-hour exercise session and a high-intensity, 1-hour exercise session caused phase shifts that were similar in magnitude and direction (55). Therefore, the dose of exercise, which is a product of intensity and duration, 
A

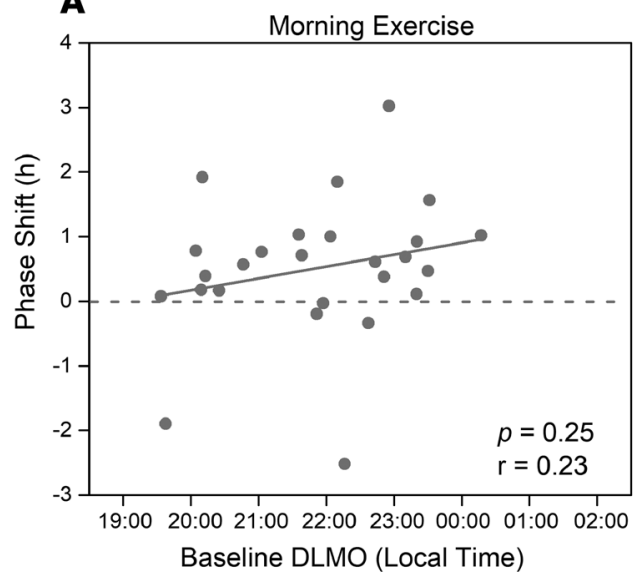

B

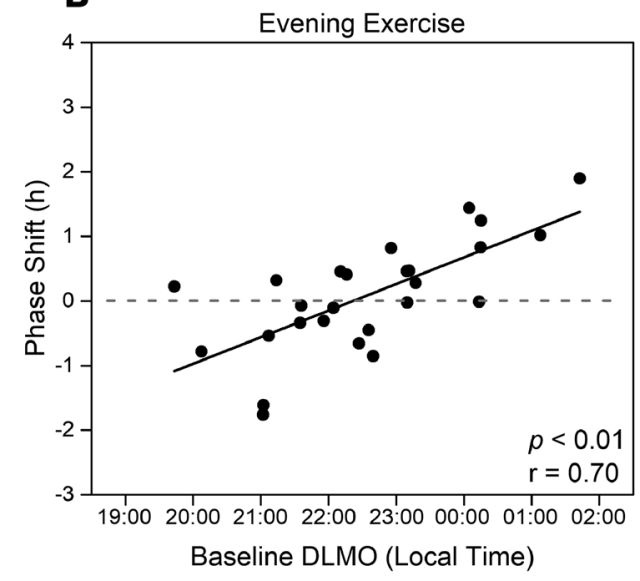

Figure 3. Phase shift is correlated with internal phase in evening exercisers. Baseline DLMO, which is marker of internal phase and a proxy for chronotype, was plotted relative to phase shift by morning $(\mathbf{A}, n=26)$ or evening $(\mathbf{B}, n=26)$ exercise. Data were analyzed by 2-tailed Pearson correlation.

may be important but could be achieved by distinct exercise regimens. We chose 30 minutes of daily, moderate-intensity cardiovascular exercise (e.g., a brisk walk or light jog) for our study because this is a feasible exercise dose that is translatable to everyday life.

Now that we have identified the proper exercise time to advance circadian rhythms, which can be tailored for chronotype, future studies should investigate the effects of timed exercise on circadian misalignment and risk factors for metabolic disorders, heart disease, and cancer. A personalized exercise intervention can be determined for an individual and could be part of a workplace wellness program; thus, it could be a widely disseminated best practice. Although it is not feasible to perform DLMOs on most individuals, chronotype questionnaires or self-report of preferred sleep timing could be used as a proxy for DLMO when prescribing the appropriate time to exercise (57). Importantly, exercising at a time of day that elicits a phase advance could increase the already-substantial benefits of exercise on health in young adults.

\section{Methods}

Participants. Data were collected between July 2017 and March 2019 in Lexington, Kentucky, USA $\left(38.0406^{\circ} \mathrm{N}, 84.5037^{\circ} \mathrm{W}\right)$, year-round, except during the 4 weeks following each semiannual daylight saving time transition. Participants were recruited via local advertisements and were first screened by telephone to query sleep habits, medical diagnoses, and medication use. Participants enrolled in the study were 18-45 years old, healthy, sedentary, and medication-free (except birth control), and they reported no psychiatric or sleep conditions. Participants had not traveled across more than 1 time zone in the 4 weeks before beginning the study and reported no night or rotating shift work in the previous year.

Study protocol. Participants were informed of the purpose and design of the study and provided written consent before participation (Figure 5). Immediately following consent, participants completed questionnaires regarding preferred daily timing of sleep and activities, or chronotype (described below). Each participant completed a Physical Activity Readiness Questionnaire (PAR-Q) and Health History Form to identify existing contraindications to exercise, as specified in the American College of Sports Medicine Guidelines for Exercise Testing and Prescription (63). At the consent session, height and weight were also collected, and participants were given actigraphy devices to wear for the duration of the study to measure activity and sleep. One to 9 days after the consent session, participants performed a baseline measure of circadian phase called DLMO to determine the timing of the internal circadian rhythm (described below). Next, cardiorespiratory fitness was determined by measuring peak oxygen consumption $\left(\mathrm{VO}_{2 \text { peak }}\right)$ from a $\mathrm{GXT}_{\max }$ as we have previously described (64). Circumference measures, as well as a dual energy x-ray absorptiometry scan (GE Lunar iDXA), were performed to assess body composition. A single, trained investigator analyzed all scans using the Lunar software Version 14.10. Participants then performed 5 days of supervised treadmill exercise in the morning or evening. Participant randomization was performed using 


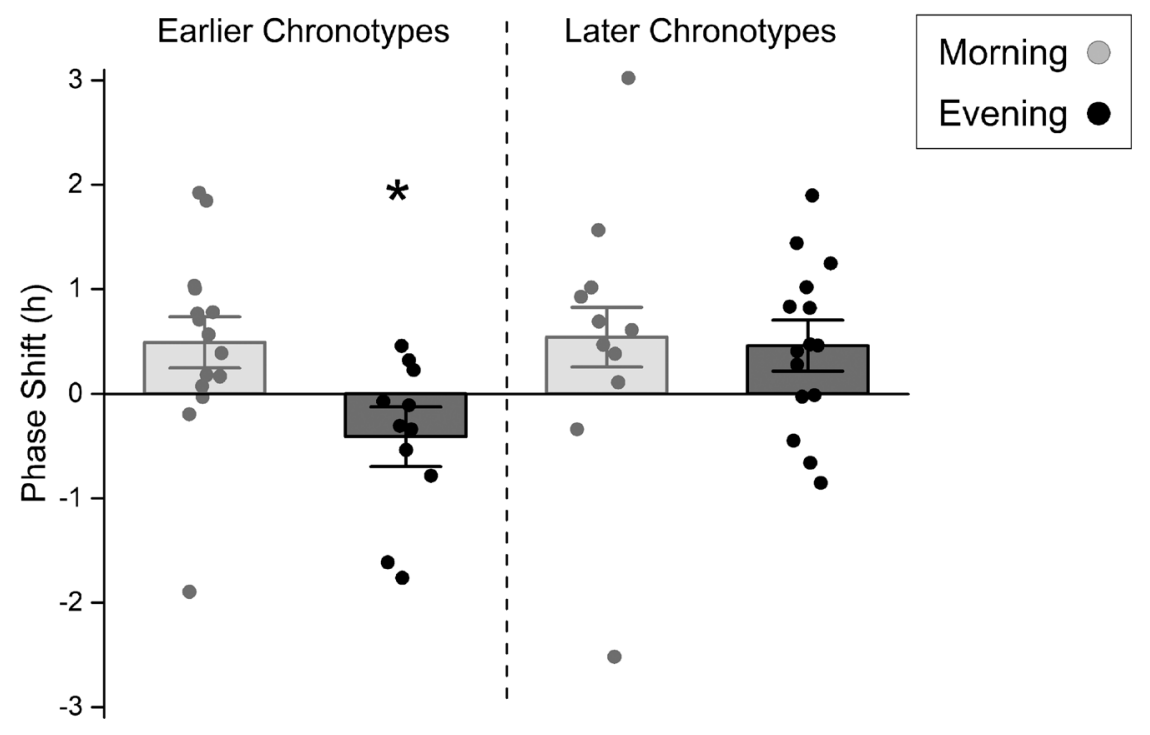

Figure 4. The effects of timed exercise on phase shifts depend on chronotype. Chronotype was dichotomized with a median split, and the effect of morning ( $n=26)$ and evening $(n=26)$ exercise on phase shift was separately analyzed in earlier $(n=26)$ and later chronotypes $(n=26)$. Data points are raw data. Bars are mean \pm SEM from 2-way ANCOVA model with exercise group and dichotomized DLMO. ${ }^{*} P<0.05$ vs. all other groups.

Statistical Analysis Software (SAS, Version 9.4). Participants maintained a heart rate corresponding to $70 \%$ $\mathrm{VO}_{2 \text { peak }}$ for 30 continuous minutes during exercise. One day after the final day of exercise, a postexercise DLMO was performed to assess changes in the internal rhythm resulting from the exercise intervention.

Chronotype questionnaires. Each participant completed the Morningness-Eveningness Questionnaire (self-assessment version, MEQ-SA) to determine chronotype (65). We also collected the Munich Chronotype Questionnaire (MCTQ) to estimate habitual bedtime (37). Previous studies have used the MCTQ to calculate $\mathrm{MSF}_{\mathrm{sc}}$ (midsleep on free days sleep corrected ) as a measure of chronotype; however, we were unable to use this measure since $42 \%$ of our participants used alarms on free days or had irregular work schedules (6).

DLMO. DLMO was performed as described previously (66). A conventional DLMO protocol was conducted, with sample collection beginning 8 hours before habitual bedtime $(67,68)$. Participants were instructed to abstain from taking nonsteroidal antiinflammatory drugs (NSAIDs) throughout the study and from eating bananas, caffeine, and chocolate and from drinking alcohol on the day of the assessment because these have been shown to alter melatonin measurements (69-72). Participants were exposed to $<10$ lux at eye level, and no light-emitting electronic devices were permitted during DLMO testing to avoid light suppression of melatonin (73). Saliva samples were collected hourly, except between 2 and 4 hours before habitual bedtime, when samples were collected every 30 minutes (because this is when melatonin levels typically rise; ref. 57), and the final sample was collected at or just after habitual bedtime. Participants were allowed to eat snacks (snacks contained no artificial colorants) except during the 30 minutes before sample collection. They were instructed to rinse with water 30 minutes and 15 minutes before sampling and to remain seated for 15 minutes before sample collection. Saliva was collected in salivettes (Sardest). Melatonin was measured by SolidPhase Inc. using a BUHLMANN Direct Saliva Melatonin Radio Immunoassay test kit. DLMO was calculated as the time when melatonin concentration exceeded and remained above $4 \mathrm{pg} / \mathrm{mL}$ (linear interpolation) (74, 75). DLMOs were performed at baseline and 1 day after the final day of exercise (Figure 5).

Actigraphy. Beginning at the time of consent, and for the duration of the study, a triaxial actigraphy device was worn on the nondominant wrist. Actigraphy data were downloaded in 10-second epochs and analyzed using AcitLife sotware (version 6.13.3). Wear-time validation was performed by indicating nonwear times in the software. Following reintegration to 60-second epochs, the Sadeh algorithm (76), combined with sleep logs, was used to assess sleep timing, duration, and sleep fragmentation (77). Participants wore the actigraphy watches for 1-9 days before the baseline DLMO, so baseline sleep data could not be analyzed. Sleep data during the 5 exercise days were analyzed. Sleep data were unavailable for 8 participants because of missing data when they removed their actigraphy devices during sleep. These 8 participants completed the study, but sleep was not analyzed in these participants.

Exercise intervention. We normalized exercise timing across participants by scheduling exercise relative to baseline DLMO. During the consent session, each participant was randomized to either morning (10 hours after DLMO) or evening (20 hours after DLMO) exercise. Participants were expected to arrive and 


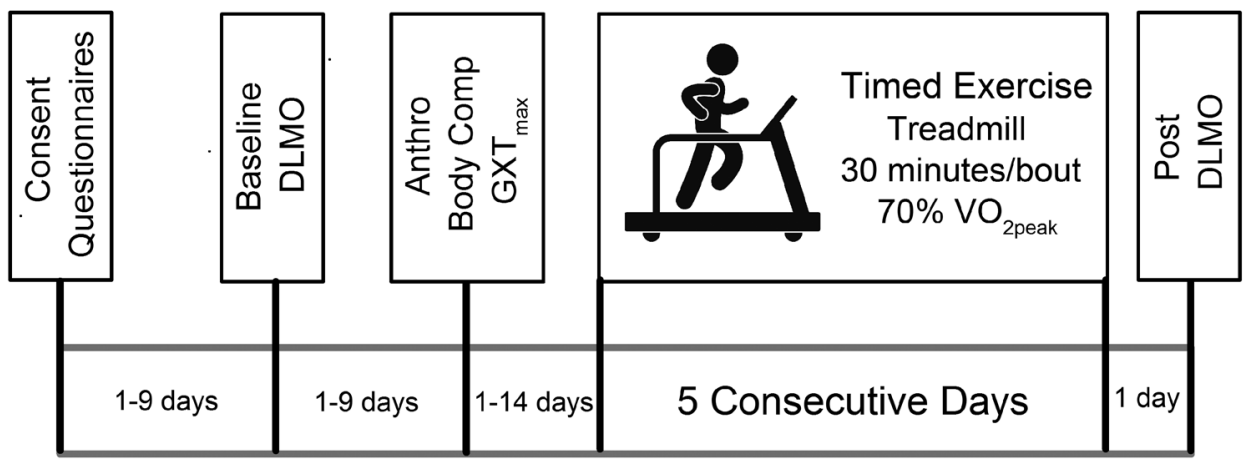

Figure 5. Study protocol. During consent, participants completed questionnaires, were measured for height and weight, and began wearing an actigraphy device. One to 9 days later, we measured baseline circadian phase with the dim light melatonin onset (DLMO) assay. Next, we took anthropometric (Anthro) and body composition (Body comp) measures and performed a maximal graded exercise test ( $\left.\mathrm{CXT}_{\text {max }}\right)$ to determine peak oxygen consumption $\left(\mathrm{VO}_{\text {2peak }}\right)$. Participants then performed 5 days of treadmill exercise, where they maintained a heart rate corresponding to $70 \%$ $\mathrm{VO}_{2 \text { peak }}$ for 30 continuous minutes, in the morning or evening. One day after the final day of exercise, postexercise circadian phase was assessed by the DLMO assay.

begin exercise within 1 hour of the scheduled time (actual exercise began $0.38 \pm 0.07$ hours from target). For 8 participants, we estimated exercise time using habitual sleep timing self-reported on the MCTQ instead of based on DLMO because the melatonin assay kit was temporarily not available (we were unable to quickly measure melatonin in those 8 participants). Those 8 participants otherwise completed the study as usual. Participants exercised for 30 minutes for 5 consecutive days at the designated time. Exercise training was performed on a treadmill, and intensity was maintained at a heart rate corresponding to $70 \%$ of $\mathrm{VO}_{2 \text { peak }}$ (determined from GXT $\mathrm{T}_{\max }$ ).

Phase shifts. One day after the final day of exercise, each participant performed a postexercise DLMO. Phase shifts were calculated as the difference (in hours) of postexercise DLMO subtracted from baseline DLMO. As is convention, phase advances were expressed as positive values and phase delays as negative values.

Statistics. Data were analyzed using SAS (Version 9.4). Descriptive data are presented as means \pm SEM. Baseline participant characteristics for the morning and evening exercise groups were compared using 2-tailed independent sample $t$ tests. Potential confounders (e.g., baseline DLMO, MEQ, and midsleep) were identified by examining bivariate associations. One- or 2-way ANCOVA was used to analyze the effect of exercise group on phase shift while adjusting for potential confounders. Given the sample size, separate 1-way ANCOVAs were performed, accounting for potential confounders. To further examine the effect of baseline circadian phase on phase shift in each exercise group, DLMO was dichotomized into earlier and later chronotype based on a median split (early chronotypes $<22: 12$ baseline DLMO; late chronotypes $\geq 22: 12$ baseline DLMO). A stratified analysis was conducted, using a 2-way ANCOVA, to estimate the phase shift difference for earlier and later chronotypes. $P<0.05$ was considered significant.

Study approval. The study was reviewed and approved by the University of Kentucky IRB, and participants provided written informed consent before inclusion in the study.

\section{Author contributions}

JMT, PAK, JLC, and JSP designed the experiments. JMT, WSB, and JLC performed the experiments. JMT, HMB, KJM, JLC, and JSP analyzed the data. JMT, PAK, JLC, and JSP wrote the manuscript. All authors provided comments and approved the manuscript.

\section{Acknowledgments}

We thank Marian Boyko, Parashar Joshi, Tori Vogler, and Jeffrey Chalfant for assistance in supervising exercise and DLMO sessions and Sarah Herricks and Rachel Reynolds for data entry and analysis. We thank Roelof Hut and Emma Wams for assistance with designing the DLMO protocol and for discussions of the study design. We thank Céline Vetter and Akram Imam for assistance with analyzing actigraphy data and for insightful discussions of the data. 
Address correspondence to: Julie S. Pendergast, 316 Thomas Hunt Morgan Building, Department of Biology, University of Kentucky, Lexington, Kentucky 40506, USA. Phone: 859.218.6770; Email: julie.pendergast@uky.edu.

1. Pittendrigh CS. Temporal organization: reflections of a Darwinian clock-watcher. Annu Rev Physiol. 1993;55:16-54.

2. DeCoursey PJ, Krulas JR. Behavior of SCN-lesioned chipmunks in natural habitat: a pilot study. J Biol Rhythms. 1998;13(3):229-244.

3. Hurd MW, Ralph MR. The significance of circadian organization for longevity in the golden hamster. J Biol Rhythms. 1998;13(5):430-436.

4. Woelfle MA, Ouyang Y, Phanvijhitsiri K, Johnson CH. The adaptive value of circadian clocks: an experimental assessment in cyanobacteria. Curr Biol. 2004;14(16):1481-1486.

5. Wright KP, McHill AW, Birks BR, Griffin BR, Rusterholz T, Chinoy ED. Entrainment of the human circadian clock to the natural light-dark cycle. Curr Biol. 2013;23(16):1554-1558.

6. Roenneberg T, Allebrandt KV, Merrow M, Vetter C. Social jetlag and obesity. Curr Biol. 2012;22(10):939-943.

7. Baron KG, Reid KJ, Wolfe LF, Attarian H, Zee PC. Phase Relationship between DLMO and Sleep Onset and the Risk of Metabolic Disease among Normal Weight and Overweight/Obese Adults. J Biol Rhythms. 2018;33(1):76-83.

8. Koo YS, et al. Outdoor artificial light at night, obesity, and sleep health: Cross-sectional analysis in the KoGES study. Chronobiol Int. 2016;33(3):301-314

9. Wong PM, Hasler BP, Kamarck TW, Muldoon MF, Manuck SB. Social Jetlag, Chronotype, and Cardiometabolic Risk. J Clin Endocrinol Metab. 2015;100(12):4612-4620.

10. Rybnikova NA, Haim A, Portnov BA. Does artificial light-at-night exposure contribute to the worldwide obesity pandemic? Int J Obes (Lond). 2016;40(5):815-823.

11. Vyas MV, et al. Shift work and vascular events: systematic review and meta-analysis. BMJ. 2012;345:e4800

12. Rutters F, et al. Is social jetlag associated with an adverse endocrine, behavioral, and cardiovascular risk profile? J Biol Rhythms. 2014;29(5):377-383.

13. Koopman ADM, et al. The Association between Social Jetlag, the Metabolic Syndrome, and Type 2 Diabetes Mellitus in the General Population: The New Hoorn Study. J Biol Rhythms. 2017;32(4):359-368.

14. Parsons MJ, et al. Social jetlag, obesity and metabolic disorder: investigation in a cohort study. Int J Obes (Lond). 2015;39(5):842-848

15. Vera B, et al. Modifiable lifestyle behaviors, but not a genetic risk score, associate with metabolic syndrome in evening chronotypes. Sci Rep. 2018;8(1):945.

16. Yu JH, et al. Evening chronotype is associated with metabolic disorders and body composition in middle-aged adults. J Clin Endocrinol Metab. 2015;100(4):1494-1502.

17. Kivelä L, Papadopoulos MR, Antypa N. Chronotype and Psychiatric Disorders. Curr Sleep Med Rep. 2018;4(2):94-103.

18. Maukonen M, et al. The associations between chronotype, a healthy diet and obesity. Chronobiol Int. 2016;33(8):972-981.

19. Baron KG, et al. Circadian timing and alignment in healthy adults: associations with BMI, body fat, caloric intake and physical activity. Int J Obes (Lond). 2017;41(2):203-209.

20. Youngstedt SD, Kline CE, Elliott JA, Zielinski MR, Devlin TM, Moore TA. Circadian Phase-Shifting Effects of Bright Light, Exercise, and Bright Light + Exercise. J Circadian Rhythms. 2016;14:2.

21. Miyazaki T, Hashimoto S, Masubuchi S, Honma S, Honma KI. Phase-advance shifts of human circadian pacemaker are accelerated by daytime physical exercise. Am J Physiol Regul Integr Comp Physiol. 2001;281(1):R197-R205.

22. Barger LK, Wright KP, Hughes RJ, Czeisler CA. Daily exercise facilitates phase delays of circadian melatonin rhythm in very dim light. Am J Physiol Regul Integr Comp Physiol. 2004;286(6):R1077-R1084.

23. Hut RA, Mrosovsky N, Daan S. Nonphotic entrainment in a diurnal mammal, the European ground squirrel (Spermophilus citellus). J Biol Rhythms. 1999;14(5):409-419.

24. Mrosovsky N, Salmon PA, Menaker M, Ralph MR. Nonphotic phase shifting in hamster clock mutants. J Biol Rhythms. 1992;7(1):41-49.

25. Hu JP, Guo YH, Wang F, Zhao XP, Zhang QH, Song QH. Exercise improves cognitive function in aging patients. Int J Clin Exp Med. 2014;7(10):3144-3149.

26. Garber CE, et al. American College of Sports Medicine position stand. Quantity and quality of exercise for developing and maintaining cardiorespiratory, musculoskeletal, and neuromotor fitness in apparently healthy adults: guidance for prescribing exercise. Med Sci Sports Exerc. 2011;43(7):1334-1359.

27. Short KR, et al. Impact of aerobic exercise training on age-related changes in insulin sensitivity and muscle oxidative capacity. Diabetes. 2003;52(8):1888-1896.

28. Bartholomew JB, Morrison D, Ciccolo JT. Effects of acute exercise on mood and well-being in patients with major depressive disorder. Med Sci Sports Exerc. 2005;37(12):2032-2037.

29. Ruscheweyh R, et al. Physical activity and memory functions: an interventional study. Neurobiol Aging. 2011;32(7):1304-1319.

30. Hunter GR, Brock DW, Byrne NM, Chandler-Laney PC, Del Corral P, Gower BA. Exercise training prevents regain of visceral fat for 1 year following weight loss. Obesity (Silver Spring). 2010;18(4):690-695.

31. Sato S, et al. Time of Exercise Specifies the Impact on Muscle Metabolic Pathways and Systemic Energy Homeostasis. Cell Metab. 2019;30(1):92-110.e4.

32. Ezagouri S, et al. Physiological and Molecular Dissection of Daily Variance in Exercise Capacity. Cell Metab. 2019;30(1):78-91.e4.

33. Edwards B, Waterhouse J, Atkinson G, Reilly T. Exercise does not necessarily influence the phase of the circadian rhythm in temperature in healthy humans. J Sports Sci. 2002;20(9):725-732.

34. Youngstedt SD, Elliott JA, Kripke DF. Human circadian phase-response curves for exercise. J Physiol (Lond). 2019;597(8):2253-2268.

35. Yamanaka $Y$, et al. Morning and evening physical exercise differentially regulate the autonomic nervous system during noctur- 
nal sleep in humans. Am J Physiol Regul Integr Comp Physiol. 2015;309(9):R1112-R1121.

36. Buxton OM, Lee CW, L'Hermite-Baleriaux M, Turek FW, Van Cauter E. Exercise elicits phase shifts and acute alterations of melatonin that vary with circadian phase. Am J Physiol Regul Integr Comp Physiol. 2003;284(3):R714-R724.

37. Roenneberg T, Wirz-Justice A, Merrow M. Life between clocks: daily temporal patterns of human chronotypes. J Biol Rhythms. 2003;18(1):80-90.

38. Lewy AJ, Sack RL. The dim light melatonin onset as a marker for circadian phase position. Chronobiol Int. 1989;6(1):93-102

39. Bredella MA. Sex Differences in Body Composition. Adv Exp Med Biol. 2017;1043:9-27.

40. Sparling PB. A meta-analysis of studies comparing maximal oxygen uptake in men and women. Res Q Exerc Sport. 1980;51(3):542-552.

41. McMahon DM, et al. Relationships between chronotype, social jetlag, sleep, obesity and blood pressure in healthy young adults Chronobiol Int. 2019;36(4):493-509.

42. Fischer D, Lombardi DA, Marucci-Wellman H, Roenneberg T. Chronotypes in the US - Influence of age and sex. PLoS One. 2017;12(6):e0178782.

43. Kantermann T, Sung H, Burgess HJ. Comparing the Morningness-Eveningness Questionnaire and Munich ChronoType Questionnaire to the Dim Light Melatonin Onset. J Biol Rhythms. 2015;30(5):449-453.

44. [No authors listed]. American College of Sports Medicine Position Stand. The recommended quantity and quality of exercise for developing and maintaining cardiorespiratory and muscular fitness, and flexibility in healthy adults. Med Sci Sports Exerc. 1998;30(6):975-991.

45. [No authors listed]. American College of Sports Medicine position statement on the recommended quantity and quality of exercise for developing and maintaining fitness in healthy adults. Med Sci Sports. 1978;10(3):vii-vix.

46. Woods RA. Sports Exercise. US BUREAU OF LABOR STATISTICS. 2017. https://www.bls.gov/spotlight/2017/sports-and-exercise/home.htm.

47. Khalsa SB, Jewett ME, Cajochen C, Czeisler CA. A phase response curve to single bright light pulses in human subjects. J Physiol (Lond). 2003;549(Pt 3):945-952.

48. Duffy JF, Czeisler CA. Effect of Light on Human Circadian Physiology. Sleep Med Clin. 2009;4(2):165-177.

49. Vetter C, et al. Association Between Rotating Night Shift Work and Risk of Coronary Heart Disease Among Women. JAMA. 2016;315(16):1726-1734.

50. Jankowiak S, et al. Current and cumulative night shift work and subclinical atherosclerosis: results of the Gutenberg Health Study. Int Arch Occup Environ Health. 2016;89(8):1169-1182.

51. Vetter C, Devore EE, Ramin CA, Speizer FE, Willett WC, Schernhammer ES. Mismatch of Sleep and Work Timing and Risk of Type 2 Diabetes. Diabetes Care. 2015;38(9):1707-1713.

52. Rubio-Sastre P, Gómez-Abellán P, Martinez-Nicolas A, Ordovás JM, Madrid JA, Garaulet M. Evening physical activity alters wrist temperature circadian rhythmicity. Chronobiol Int. 2014;31(2):276-282.

53. Youngstedt SD, Kripke DF, Elliott JA. Circadian phase-delaying effects of bright light alone and combined with exercise in humans. Am J Physiol Regul Integr Comp Physiol. 2002;282(1):R259-R266.

54. Van Reeth $\mathrm{O}$, et al. Nocturnal exercise phase delays circadian rhythms of melatonin and thyrotropin secretion in normal men. Am J Physiol. 1994;266(6 Pt 1):E964-E974.

55. Buxton OM, Frank SA, L'Hermite-Balériaux M, Leproult R, Turek FW, Van Cauter E. Roles of intensity and duration of nocturnal exercise in causing phase delays of human circadian rhythms. Am J Physiol. 1997;273(3 Pt 1):E536-E542.

56. Eastman CI, Hoese EK, Youngstedt SD, Liu L. Phase-shifting human circadian rhythms with exercise during the night shift. Physiol Behav. 1995;58(6):1287-1291.

57. Burgess HJ, Eastman CI. The dim light melatonin onset following fixed and free sleep schedules. J Sleep Res. 2005;14(3):229-237.

58. Sletten TL, Vincenzi S, Redman JR, Lockley SW, Rajaratnam SM. Timing of sleep and its relationship with the endogenous melatonin rhythm. Front Neurol. 2010;1:137.

59. Baehr EK, Eastman CI, Revelle W, Olson SH, Wolfe LF, Zee PC. Circadian phase-shifting effects of nocturnal exercise in older compared with young adults. Am J Physiol Regul Integr Comp Physiol. 2003;284(6):R1542-R1550.

60. Sharma M, et al. Circadian rhythms of melatonin and cortisol in aging. Biol Psychiatry. 1989;25(3):305-319.

61. Duffy JF, Zitting KM, Chinoy ED. Aging and Circadian Rhythms. Sleep Med Clin. 2015;10(4):423-434.

62. Saeed Y, Abbott SM. Circadian Disruption Associated with Alzheimer's Disease. Curr Neurol Neurosci Rep. 2017;17(4):29.

63. Riebe D, Ehrman JK, Liguori G, Magal M. ACSM's guidelines for exercise testing prescription. Indianapolis,IN; American College of Sports Medicine: 2018.

64. Tranel HR, et al. Physical activity, and not fat mass is a primary predictor of circadian parameters in young men. Chronobiol Int. 2015;32(6):832-841

65. Horne JA, Ostberg O. A self-assessment questionnaire to determine morningness-eveningness in human circadian rhythms. Int $J$ Chronobiol. 1976;4(2):97-110.

66. Burgess HJ, Wyatt JK, Park M, Fogg LF. Home Circadian Phase Assessments with Measures of Compliance Yield Accurate Dim Light Melatonin Onsets. Sleep. 2015;38(6):889-897.

67. Woelders T, Beersma DGM, Gordijn MCM, Hut RA, Wams EJ. Daily Light Exposure Patterns Reveal Phase and Period of the Human Circadian Clock. J Biol Rhythms. 2017;32(3):274-286.

68. Wams EJ, et al. Linking Light Exposure and Subsequent Sleep: A Field Polysomnography Study in Humans. Sleep. 2017;40(12):zsx165.

69. Wright KP, Badia P, Myers BL, Plenzler SC, Hakel M. Caffeine and light effects on nighttime melatonin and temperature levels in sleep-deprived humans. Brain Res. 1997;747(1):78-84.

70. Rupp TL, Acebo C, Carskadon MA. Evening alcohol suppresses salivary melatonin in young adults. Chronobiol Int. 2007;24(3):463-470

71. Sae-Teaw M, Johns J, Johns NP, Subongkot S. Serum melatonin levels and antioxidant capacities after consumption of pineapple, orange, or banana by healthy male volunteers. J Pineal Res. 2013;55(1):58-64.

72. Murphy PJ, Myers BL, Badia P. Nonsteroidal anti-inflammatory drugs alter body temperature and suppress melatonin in 
humans. Physiol Behav. 1996;59(1):133-139.

73. Benloucif S, et al. Measuring melatonin in humans. J Clin Sleep Med. 2008;4(1):66-69.

74. Saxvig IW, et al. Objective measures of sleep and dim light melatonin onset in adolescents and young adults with delayed sleep phase disorder compared to healthy controls. J Sleep Res. 2013;22(4):365-372.

75. Crowley SJ, Suh C, Molina TA, Fogg LF, Sharkey KM, Carskadon MA. Estimating the dim light melatonin onset of adolescents within a 6-h sampling window: the impact of sampling rate and threshold method. Sleep Med. 2016;20:59-66.

76. Sadeh A, Sharkey KM, Carskadon MA. Activity-based sleep-wake identification: an empirical test of methodological issues. Sleep. 1994;17(3):201-207.

77. Loewen A, Siemens A, Hanly P. Sleep disruption in patients with sleep apnea and end-stage renal disease. J Clin Sleep Med. 2009;5(4):324-329. 